\title{
Rôle De La Fracturation Dans La Circulation Des Eaux Souterraines Et La Répartition Des Sources : Cas Du Bassin Hydrogéologique De Sefrou (Moyen Atlas Septentrional, Maroc)
}

\begin{abstract}
Bouhsine EI Fellah Idrissi
Laboratoire des Sciences de la Vie et de la Terre, Centre Régionale des Métiers d'Education et de Formation, Route Quartier Al Matar El Jadida, \& Laboratoire de Géosciences Marines et Sciences du Sol, Université Chouaib

Doukkali, Faculté des Sciences, Avenue de Facultés, El Jadida - Maroc

\section{Said Hinaje}

Laboratoire de Géo Ressources et Environnement, Université Sidi Mohammed Ben Abdallah, Faculté des Sciences et Techniques, Fès-Saïs, Maroc

\section{Bouamama Cherai}

Département des Sciences de la Vie et de la Terre, Centre Régionale des Métiers d'Education et de Formation, Tanger - Tétouan

\section{Khalid Mehdi}

Laboratoire Géosciences Marines et Sciences du Sol, Université Chouaib

Doukkali, Faculté des Sciences, Avenue de Facultés, El Jadida - Maroc
\end{abstract}

\begin{abstract}
The formation of the basin of Sefrou is related to the normal successive movements of the faults N130, N80, N30 and N170 which follow one another since the upper Miocene until the recent medium-Quaternary. Seeing the dynamic of groundwater in the carbonated lands (Liasic dolomites) is primarily guided by the fractures and the cracks; we propose to study the influence of brittle tectonics on the spatial organization of the flows and the relation of the groundwater circulation with the fracturing. The analysis of the fracturing (affecting the Flavio-lake and travertines deposits of plio-quaternary age and their Miocene and Jurassic substratum) and the structural cuts as well as the correlation of the piezometric map established from the piezometric reports with that of the fracturing, allowed us to note that the fractures play a very important part in the geometry of the Liasic aquifer and the mode of the circulation of groundwater. This circulation is dependent on the zones of weaknesses along the faults and fractures. Water
\end{abstract}


emergences are very variable and controlled by the paramount influence of the nature of the lithological formations and the tectonic structure on underground circulations. Indeed, the sources of water located in the basin of Sefrou are indexed, according to their origin, inside two types: sources of emergence and sources of discharge.

Keywords: Moroccan Middle Atlas, Sefrou basin, brittle tectonic, Fracturing, groundwater circulation, sources

\section{Résumé}

La formation du bassin de Sefrou est liée aux jeux normaux successifs des failles N130, N80, N30 et N170 qui se succèdent depuis le Miocène supérieur jusqu'au Quaternaire moyen-récent. Etant donné que la dynamique des eaux souterraines dans les terrains carbonatés (dolomies liasiques) est essentiellement guidée par les fractures et les fissures; nous nous proposons d'étudier l'influence de la tectonique cassante sur l'organisation spatiale des écoulements et la relation de la circulation des eaux souterraines avec la fracturation. L'analyse de la fracturation (affectant les dépôts fluvio-lacustres et travertinaux d'âge plio-quaternaire et leurs substratums miocène et jurassique) et des coupes structurales réalisées ainsi que la corrélation de la carte piézométrique établie à partir des relevées piézométriques avec celle de la fracturation, nous ont permis de constater que les fractures jouent un rôle très important dans la géométrie de l'aquifère liasique et le mode de la circulation des eaux souterraines. Cette circulation est tributaire des zones de faiblesses le long des failles et fractures. Les émergences d'eaux sont très variables et contrôlées par l'influence primordiale de la nature des formations lithologiques et de la structure tectonique sur les circulations souterraines. En effet, les sources d'eau repérées dans le bassin de Sefrou sont répertoriées, selon leur origine, à l'intérieur de deux types : sources d'émergence et sources de déversement.

Mots clés : Moyen Atlas marocain, Bassin de Sefrou, Tectonique cassante, Fracturation, Circulation des eaux souterraines, sources

\section{Introduction}

Le bassin de Sefrou par sa situation, ses constitutions lithologiques et ses structures, joue un rôle important en hydrogéologie de la zone. La présence de l'Oued Aggay qui draine celui-ci, ainsi que de nombreuses sources débitant des quantités considérables d'eaux, témoignent de cette importance. Cependant, ses caractéristiques lithologiques et structurales en font un système hydrogéologique complexe, où les dépôts liasiques jouent un rôle prédominant. Ces dépôts sont constitués essentiellement par une 
puissante série carbonatée, généralement formée de dolomies bréchiques d'âge Lias inférieur. Cette couche aquifère renferme une importante nappe d'eau qui est retenue par un plancher imperméable constitué d'argilites rouges du Trias supérieur-Lias inférieur (Cousminer \& Manspeizer, 1976 ; Biron \& Courtinat, 1982 ; Baudelot et al., 1986 ; Sabaoui, 1987). Toutefois on constate d'autres circulations de celle-ci au niveau des basaltes tholeiitique de Mimet et de Bhalil, situées au NW et à l'W de ce bassin. Ces basaltes d'âges Trias supérieur - Lias inférieur affleurent dans des boutonnières au $\mathrm{N}$ et au NW du Causse moyen atlasique, à la faveur de zones faillées.

La tectonique cassante polyphasée, par le jeu des accidents et des failles, compartimente les différents aquifères. Cette tectonique a engendré une géométrie complexe avec des blocs faillés limitant l'extension des couches aquifères.

L'étude de l'influence de la tectonique cassante sur la circulation des eaux souterraines et de surface dans le bassin de Sefrou n'a fait l'objet d'aucun travail académique récent. Les anciens travaux à caractère hydrogéologique qui ont touché le Moyen Atlas sont ceux de Margat, (1952) ; Taltasse, (1952) ; Bentayeb \& Leclerc, (1977). Etant donné que la dynamique des eaux souterraines et de surface dans les terrains carbonatés est essentiellement guidée par les fractures et les fissures ; nous nous proposons d'étudier :

- l'apport de l'analyse de la fracturation à la caractérisation de la géométrie et de l'évolution des couches réservoirs ;

- la détermination de l'influence de la tectonique cassante sur l'organisation spatiale des écoulements et la relation de la circulation des eaux souterraines avec la fracturation.

L'importance du rôle hydrogéologique des structures cassantes identifiées (failles, horsts, grabens), permet à l'échelle d'un massif, de sélectionner les zones favorables ou défavorables à l'implantation de captages d'eau (puits, forages).

Le bassin de Sefrou qui fait l'objet de notre étude correspond à la partie occidentale du Causse de Sefrou. Il appartient à la zone de transition entre deux domaines structuraux : le Moyen Atlas au Sud et le sillon sudrifain au Nord (Fig. 1). Il est limité au N par la plaine de Saïs qui présente une couverture formée de terrains néogène et de quaternaire, à l'E par l'accident de Tizi N'Tretten (A.T.T) qui le sépare de la partie oriental du causse de Sefrou (plateau D’Azzaba - El Ouata), au S par la faille de J.Kandar qui le sépare du causse d'Imouzzer et à l'Ouest par l'accident de Dayet Aoua qui forme approximativement la limite avec la plaine de Saïs (Fig. 2). 


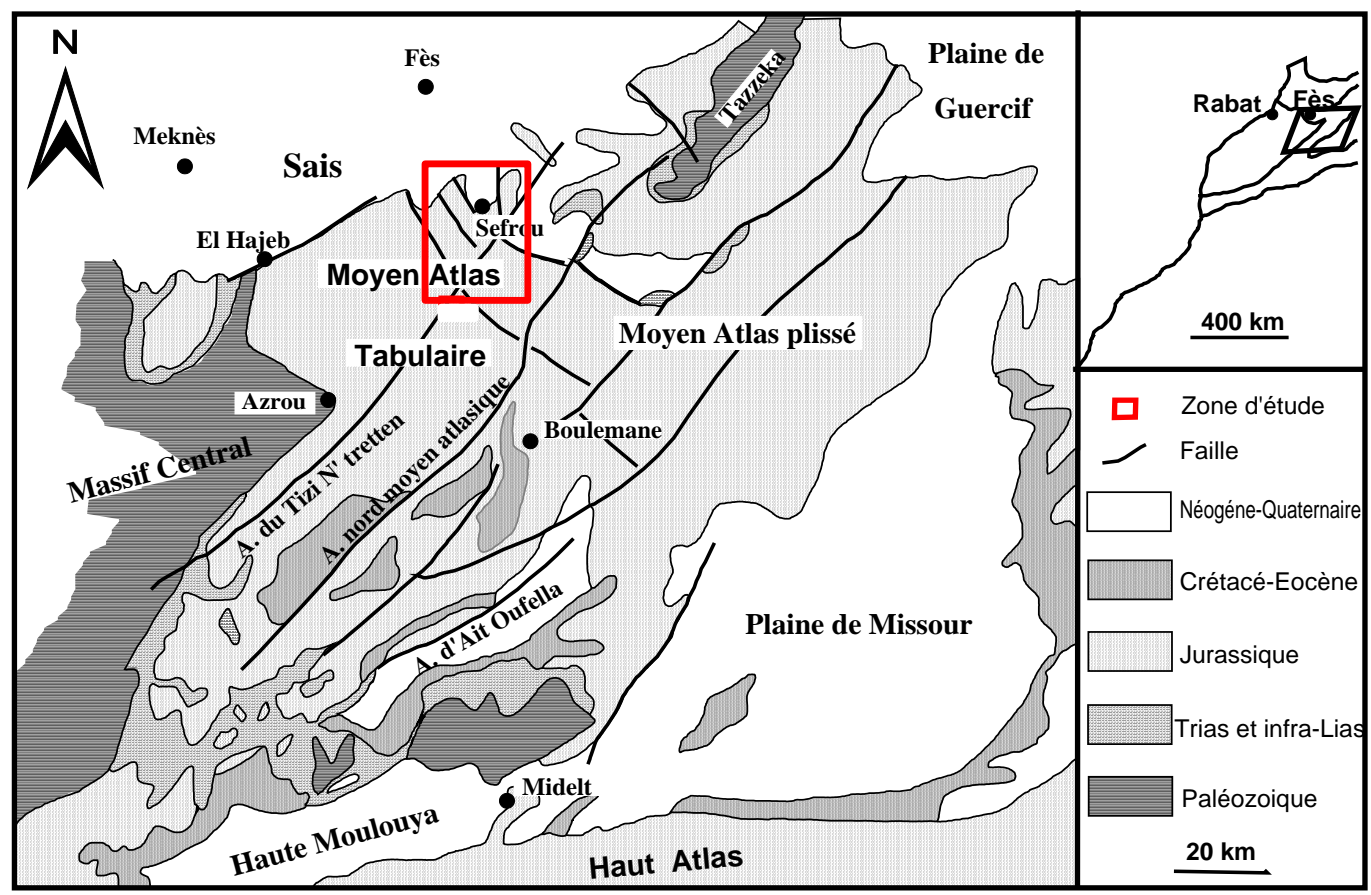

Figure 1 : Carte géologique simplifiée du Moyen Atlas (d’après la carte géologique du Maroc au 1/1000000 ; modifiée).

\section{Matériels et méthodes}

Notre étude consiste en une approche montrant l'importance hydrogéologique des fractures identifiées en fonction de leur nature et de leur orientation. La méthodologie adoptée pour atteindre cet objectif est basée sur :

- $\quad$ L'analyse de la déformation cassante, afin de déterminer l'évolution des paléochamps de contraintes (du Miocène supérieur jusqu'à l'actuel), et leur relation avec la genèse et l'évolution du bassin hydrogéologique de Sefrou et des différentes unités structurales qui les influencent. La chronologie des épisodes tectoniques est basée sur l'âge des formations, sur les recoupements des structures, sur la superposition des stries sur les miroirs des failles, sur la présence de fractures parallèles à jeux incompatible et sur la présence des structures tectoniques et microtectoniques synsédimentaires. Ce dernier facteur est primordial pour caractériser la déformation pendant une période précise ;

- le repérage et l'inventaire des points d'eau qui sont en général des puits et des sources destinés à l'alimentation et/ou à l'irrigation. Afin d'étudier la piézométrie de la nappe phréatique du bassin de Sefrou, il s'est avéré important de réaliser des cartes piézométriques. Ces dernières sont les documents de base de l'analyse, et de la schématisation des fonctions capacitives et conductrices du réservoir et du comportement 
hydrodynamique de l'aquifère. C'est la synthèse la plus importante d'une étude hydrogéologique (Castany, 1982). A Cet égard, nous avons réalisé une campagne de relevés piézométriques. Cette campagne a porté sur les puits captant la nappe phréatique au niveau de l'aquifère triasico-liasique et l'aquifère liasique, et couvre le bassin.

- $\quad$ le traitement des différentes données récoltées du terrain et de la carte géologique de la région de Sefrou qui est éditée par le Services Géologique du Maroc [SGM] (1989). Ceci nous permet d'élaborer la carte structurale et piézométrique de la zone d'étude, ainsi que l'établissement de plusieurs coupes structurales.

\section{Résultats et discussion}

Analyse de la fracturation et détermination des contraintes néogènes et quaternaires responsables de la genèse et de l'évolution du bassin hydrogéologique de Sefrou

Le bassin de Sefrou est le siège d'une activité tectonique polyphasée au Néogène et au Quaternaire (Charrière, 1990 ; Aït Brahim, 1992 ; Sabaâoui \& Hinaje, 2000 ; Hinaje et al, 2002). La formation de ce bassin est liée à quatre épisodes extensifs appartenant aux phases tectoniques qui se succèdent depuis le Miocène supérieur jusqu’au Quaternaire moyen-récent (cf.Fig. 2).

\section{Episode extensif d’âge Miocène supérieur}

Cet épisode correspond à une extension NE-SW dont l'axe $\sigma_{1}$ est vertical et l'axe $\sigma_{3}$ est horizontal. Ce régime extensif a donné naissance aux failles normales de direction moyenne N130, telles que la faille de Kandar, la faille de Bni Mellala (Faille de Aïn Dik) et la faille de 


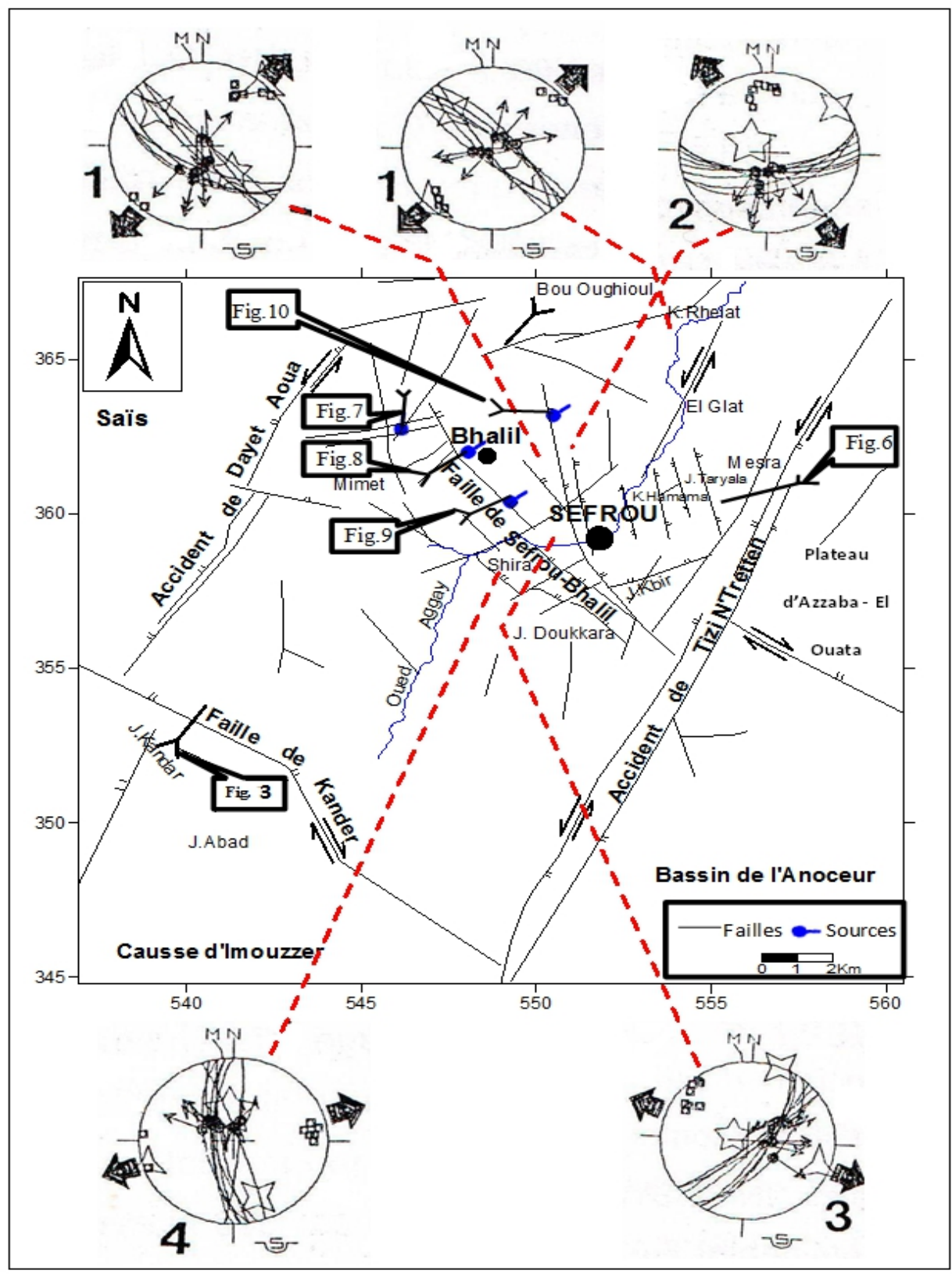

Figure 2 : Carte structurale du bassin de Sefrou et des zones avoisinantes avec les paléochamps de contraintes générateurs des effondrements tectoniques depuis le Miocène supérieur jusqu'au Quaternaire récent. 1- Episode extensif du Miocène supérieur ; 2Extension du Pliocène moyen-supérieur ; 3- Episode extensif du Quaternaire ancien-moyen ; 4- Episode extensif du Quaternaire moyen-récent.

Sefrou - Bhalil. En effet, cette tectonique extensive est responsable de la structuration du bassin de Sefrou en bloc étagés à effondrement NE depuis Jbel Kandar jusqu’à Bou Oughioul (Fig. 3). 


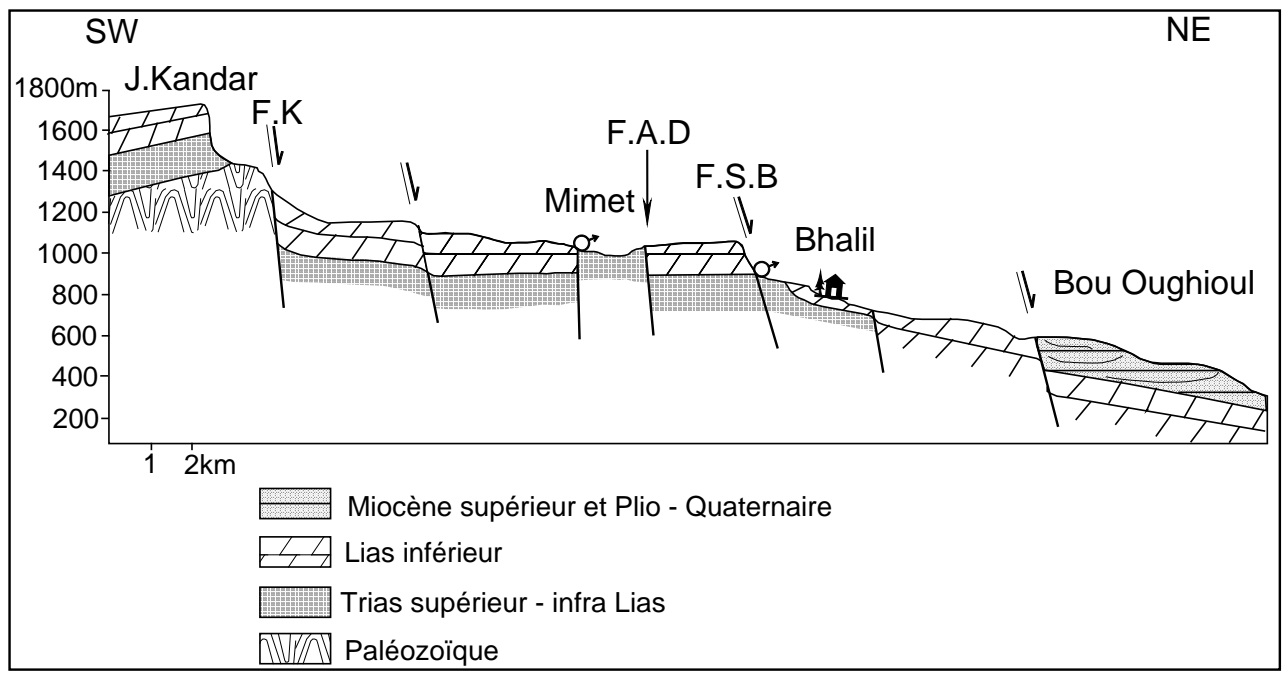

Figure 3 : Coupe structurale le long du bassin de Sefrou montrant l'effondrement vers le NE (situation de la coupe voir Fig. 2). F.A.D : faille de Aïn Dik ; F.S.B : faille de Sefrou -

Bhalil.

\section{Episode extensif d'âge Pliocène moyen - supérieur}

$\mathrm{Au}$ Pliocène moyen-supérieur le deuxième épisode extensif est caractérisé par un axe $\sigma_{3}$ orienté NNW-SSE. Cet épisode est responsable de la formation d'une seconde zone effondrée orientée ENE-WSW à E-W. Cette zone allongée de Bhalil à l'Ouest vers la zone d'El Glat à l'Est, est encadrée par des failles normales de direction N60 à N90 comme la faille de Jbel Kbir et la faille de Jerf Lahmer. Ces dernières effondrent le Lias inférieur et le Miocène supérieur vers le centre du graben, où s'installe un bassin fluvio-lacustre et travertineux. Du côté ouest, ce bassin est limité par le relief liasique formé par la faille de Sefrou - Bhalil. Celle-ci est responsable des résurgences d'eau douce qui ont donné la masse travertineuse de Jbel Binna.

\section{Episode extensif d'âge Quaternaire ancien-moyen}

Cet épisode extensif correspond à un régime de paléo-contraintes tel que l'axe $\sigma_{3}$ est horizontal et orienté N120. Cette extension est matérialisée par des failles normales NNE-SSW à NE-SW. En effet, ces failles sont responsables de la genèse d'un bassin continental fluvio-lacustre et travertineux orienté en moyenne N30. Celui-ci se superpose au bassin continental de direction moyenne N80 d'âge Pliocène moyen-supérieur (graben), et au bassin marin orienté N130 d'âge Miocène supérieur (hémigraben). Ce bassin est limité vers le Nord et vers le NW par des failles normales d'orientation NNE-SSW à NE-SW, comme la faille de Jbel Binna, La faille de Koudiat Rhellat, la faille d'Igurazène et la faille de Bou Jrou. Du 
côté est et ouest, le bassin de Sefrou est encadré respectivement par l'accident de Tizi N'Trettent et celui de Dayet Aoua. Ces deux accidents orientés N30 à N40, présentent au Quaternaire ancien-moyen, des jeux normaux à effondrement NW.

\section{Episode extensif d’âge Quaternaire moyen - récent}

Ce dernier épisode tectonique correspond à une extension N80 d’âge Quaternaire moyen - récent (Hinaje, 2004), responsable du jeu normal des failles subméridiennes. Ces failles provoquent l'installation du bassin subsident de direction N-S, où est située la ville de Sefrou (Hinaje et al., 2002). Il est comblé par une sédimentation continentale de type fluviolacustre

et travertineux. Ainsi, de l'Ouest vers l'Est de ce bassin, on peut dénombrer sept failles normales majeures, orientées N150 à N10. Ces failles ont influencé la répartition des faciès sédimentaires du Quaternaire moyen récent. Parmi ces failles, il y a celle d’El Mers - caserne militaire, de Kalaâ, de Kodiat Hamama et de Taryala. L'association de ces failles normales subméridiennes avec les failles normales antérieures, de directions moyennes N130, N80 et N30, a déterminé la genèse et le tracé hydrographique de la vallée actuelle de l'Oued Aggay, la formation et la contribution à l'évolution morpho-sédimentaire du bassin effondré de Sefrou (Hinaje, 2004).

\section{Relation entre la circulation des eaux souterraines et la fracturation}

L'hydrodynamisme des milieux fracturé reste très mal connu et n'est que très rarement rapporté aux fractures tectoniques (Bruel et al ; 1999). Les fractures contrôlent l'écoulement de l'eau souterraine et superficielle, et jouent un rôle primordial dans la productivité des réservoirs fracturés. C’est pour cela que les études hydrogéologiques doivent être appuyées par une analyse tectonique et microtectonique de la fracturation régionale.

La caractérisation hydrodynamique d'un réservoir fracturé a été souvent abordée en adoptant une vision globale des écoulements (Loiseau, 1987 ; Cacas \& De Marsily, 1987 ; Pusch \& Hokamark, 1991), par la détermination des axes des écoulements mis en parallèle avec la caractérisation géostatistique du réseau de fractures. Les principales anomalies structurales du réservoir se traduisant dans la morphologie de la surface piézométrique, sont les variations de la section, la surface du substratum et les accidents tectoniques (Castany, 1982).

Structures tectoniques influençant la circulation des eaux dans le bassin de Sefrou

Circulations des eaux souterraines dans les grabens et les horsts quaternaires 
$\mathrm{Au}$ niveau de ce bassin, on a pu mettre en évidence la présence de quatre familles de failles de direction moyenne N130, N80, N30 et N170 responsables de la structure et de la morphologie actuelle. Ces familles de failles dont le premier jeu est normal, ont permis au Moyen Atlas septentrional de surplomber le couloir Sud-rifain au Nord d'une part, et d'acquérir une structure en blocs tectoniques étagés d'autre part. Ceci va engendrer une circulation des eaux souterraines spécifique conditionnée par la structure du système aquifère.

L'analyse de la carte piézométrique que nous avons établie pour la campagne de Juillet-Août 2003 (Fig. 4) nous a permis de distinguer :

- l'allure des isopièzes de l'ensemble du bassin de Sefrou est compatible avec la structure de celui-ci. Ces isopièzes sont parallèles avec le passage de la faille N140 de Kandar, de Sefrou - Bhalil, des failles N60 à N90 et de l'accident N30 de Dayet Aoua. Au niveau de ces failles les écoulements de la nappe sont généralement perpendiculaires à celles-ci, matérialisant ainsi une transition des réservoirs soulevés vers les réservoirs effondrés ;

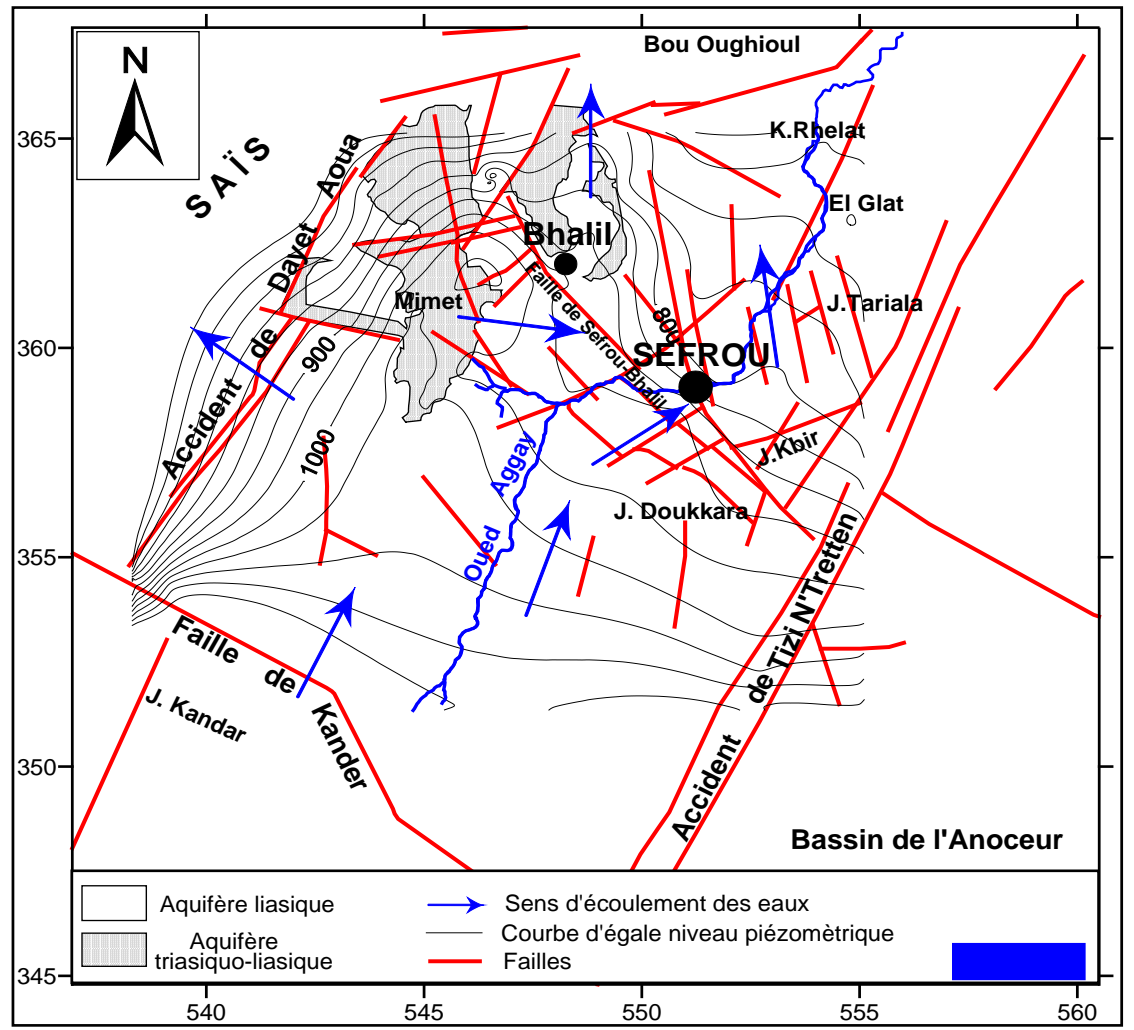

Figure 4 : Carte piézométrique du bassin de Sefrou (Juillet et Août 2003) superposée avec celle de la fracturation. 
- l’écoulement de la nappe du Sud vers le Nord se fait sous l'effet d'un compartimentage tectonique en blocs étagés. Ce compartimentage est le résultat du jeu normal effondrant vers le NE de la famille de failles N130 telle que la faille de Kandar, de Aïn Dik et de Sefrou - Bhalil. En se dirigeant vers le Nord, au niveau de la cuvette de Sefrou l'écoulement de la nappe s'oriente et converge vers celle-ci où il devient dans certaines zones orienté E-W. Ceci est dû au fait que la zone de Sefrou correspond à un bassin subsident suite à la succession des épisodes extensifs d’âges Miocène supérieur, Pliocène moyen-supérieur et Quaternaires. Ces épisodes qui ont donné naissance à des failles normales N130, N60 à N90, N30 à N45 et N170 à subméridiennes, génèrent des grabens superposés où s’installent des bassins fluvio-lacustres et travertineux. C'est cette superposition qui a amplifiée la subsidence du bassin de Sefrou qui dépasse les 200m au centre par rapport à sa périphérie (J. Kebir). Par conséquence, vu la puissance des dépôts continentaux plio-quaternaires comblant ce bassin, la nappe d'eau liasique se trouve à des profondeurs très importantes ;

- la cuvette de Sefrou correspond à un bassin hydrogéologique semi fermé, bordée à l'Est, à l’Ouest et au Sud par des affleurements liasiques qui ont un pendage général vers le centre de ce bassin. Tandis que vers le Nord, elle est ouverte sous l'effet du plongement en blocs basculés des dolomies liasiques sous les dépôts miocènes, qui les recouvrent en discordance. Ce plongement guide en effet l'écoulement de la nappe vers le Nord ;

- sur les flancs N et NW du bassin de Sefrou, on constate un déplacement généralisé de la nappe vers le Nord et le NW en direction de Saïs et du couloir sud-rifain. Ce déplacement est guidé principalement par le basculement des couches triasico-liasiques et liasiques, qui se traduit par le rapprochement des lignes isopièzes enregistrées sur la carte piézométrique (El Fellah Idrissi et al., 2005). Ce basculement est lié aux jeux normaux des failles N70 et de l'accident de Dayet Aoua orienté N30. Ces failles à effondrement vers le Nord et le NW, provoquent le dégorgement partiel de la nappe aquifère du Trias supérieur-Lias inférieur (basaltes doléritiques) et du Lias inférieur (dolomies), et la mise en charge de la nappe captive et de la nappe phréatique de la plaine de Saïs (Fig. 5). Au niveau de ces flancs la nappe du bassin de Sefrou dégorge en de nombreuses sources (Aïn Dik, Aïn Zeribir, Aïn Zeraoua....), tandis qu'au contact de ce bassin avec la plaine de Saïs la nappe alimente en profondeur la nappe captive contenue dans les dolomies liasiques et dans les horizons perméables miocènes (molasses, grés...), et en sub-surface la nappe phréatique des calcaires lacustres et des conglomérats d’âge plio-quaternaire. 


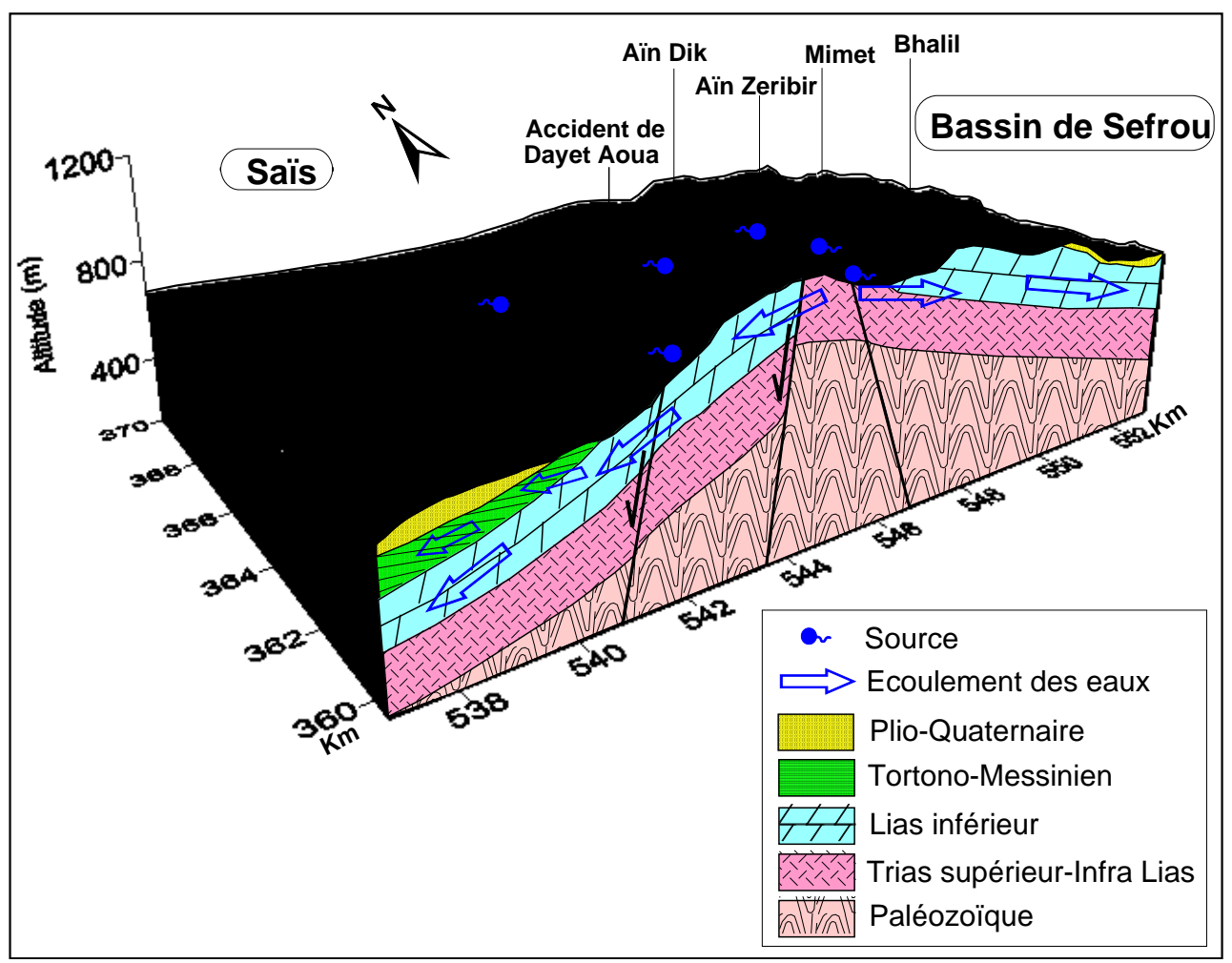

Figure 5 : Bloc diagramme schématique illustrant la relation hydrogéologique entre le bassin de Sefrou et le bassin de Saïs.

\section{Identification des axes de drainage et des structures barrières}

La circulation des eaux de la nappe en direction des compartiments affaissés est favorisée, en plus de la porosité en grand de l'aquifère dolomitique, par les failles jouant le rôle d'axes préférentiels de cheminement des eaux. En outre, les déviations des écoulements de la nappe sont dus aux différentes structures tectoniques (failles, grabens, horsts, affleurement des agriles) qui affectent la couche aquifère.

Les écoulements des eaux de la nappe selon la direction NE-SW, c'està-dire vers la zone affaissée de Sefrou - Bhalil, sont conformes avec la direction des failles NE-SW transverses par rapport à la faille de Sefrou Bhalil (N140) qui est responsable de cet affaissement (cf. Fig. 4). Ces failles à jeu décrochant senestre au Quaternaire moyen-récent, provoquent le décalage de celle-ci. Leur caractère décrochant abouti à la formation de zones bréchifiées favorisant les écoulements des eaux. Le passage de ces failles est souligné dans certains endroits par l'apparition de sources pérennes comme le cas de la source de Sidi Ali Bou Serghine à l'Ouest de Sefrou et celle de Aïn Ghzale à l’Ouest de Bhalil. Ces sources émergent au piémont des reliefs liasiques et au niveau des éboulis du Lias, de préférence 
dans les zones d'intersection de ces failles (NE-SW) avec la faille N140 de Sefrou - Bhalil. L'une de ces failles décrochantes est responsable de l'émergence de plusieurs sources alimentant l’Oued Aggay, c'est le cas de la source Aïn Ghazi. Cette faille contribue avec d'autres au traçage de la vallée de l'Oued Aggay.

Les failles décrochantes NE-SW constituent des zones de faiblesses où l'érosion est plus active. Elles constituent des endroits privilégiés pour le développement des ravins. Le plus souvent, ces failles sont parcourues par de longs talwegs constituant des axes de rassemblement et de canalisation des eaux pluviales. Ceci favorise l'infiltration d'une grande partie des eaux permettant l'alimentation de la nappe et des sources. Ces failles décrochantes NE-SW dont le jeu senestre et d’âge Quaternaire moyen-récent, affectent à la fois les basaltes triasico-liasiques, les dolomies liasiques, et les dépôts néogènes et quaternaires du bassin de Sefrou en particulier, et du Moyen et Haut Atlas en général (Hinaje, 2004). Elles assurent une large connexion entre les compartiments soulevés et affaissés par la faille de Sefrou - Bhalil. Ainsi au niveau du bassin subsident de Sefrou, ces failles participent à l'alimentation de la nappe profonde liasique et de la nappe quaternaire. Vers le NW de Sefrou, ce type de failles permettent un échange d'eau entre le réservoir basaltique triasico-liasique de Mimet et les réservoirs de Bhalil (dolomies liasiques et basaltes triasico-liasiques). L’utilisation préférentielle de ces failles décrochantes assure d'importante circulation latérale des eaux permettant ainsi l'alimentation (ou drainage) de ces réservoirs.

Au niveau du bassin subsident néogène et quaternaire de Sefrou, une autre famille de failles peut jouer aussi le rôle des axes préférentiels pour le drainage des eaux. Il s’agit des failles normales subméridiennes (El Fellah Idrissi et al., 2005) qui sont liées à l’épisode tectonique distensif d’âge Quaternaire moyen-récent, où la contrainte maximale $\sigma_{1}$ est verticale et la contrainte minimale $\sigma_{3}$ est horizontale et orientée N80. Cette extension provoque un allongement au niveau des failles subméridiennes et des fissures proches de cette direction. En effet, l'ouverture de ces fractures est bien visible à Jbel Binna (bordure Ouest) au niveau des calcaires lacustres, et à Jbel Tariala (bordure est du bassin) au niveau des dolomies liasiques. A Jbel Tariala, une faille subméridienne à pendage Ouest, présente à la surface une ouverture qui peut atteindre $2 \mathrm{~m}$ de large. Au niveau de cette localité les dolomies sont affectées par une intense fracturation provoquant la formation d'une doline sur le flanc ouest de celui-ci.

Le jeu normal des failles subméridiennes aboutit à la formation des horsts de Kodiat Hamama, de Tariala et de Tagnit, ainsi que la création des grabens subméridiens tels que celui où s’installe la ville de Sefrou (Fig. 6). Ces horsts constituent la limite orientale du bassin subsident de Sefrou et peuvent influencés la direction de cheminement des eaux qui suivent 
habituellement le sens des zones d'effondrements. Ainsi, la majorité des écoulements se fait vers ces grabens qui canalisent la grande partie des eaux et guident le déplacement de la nappe liasique vers le Nord.

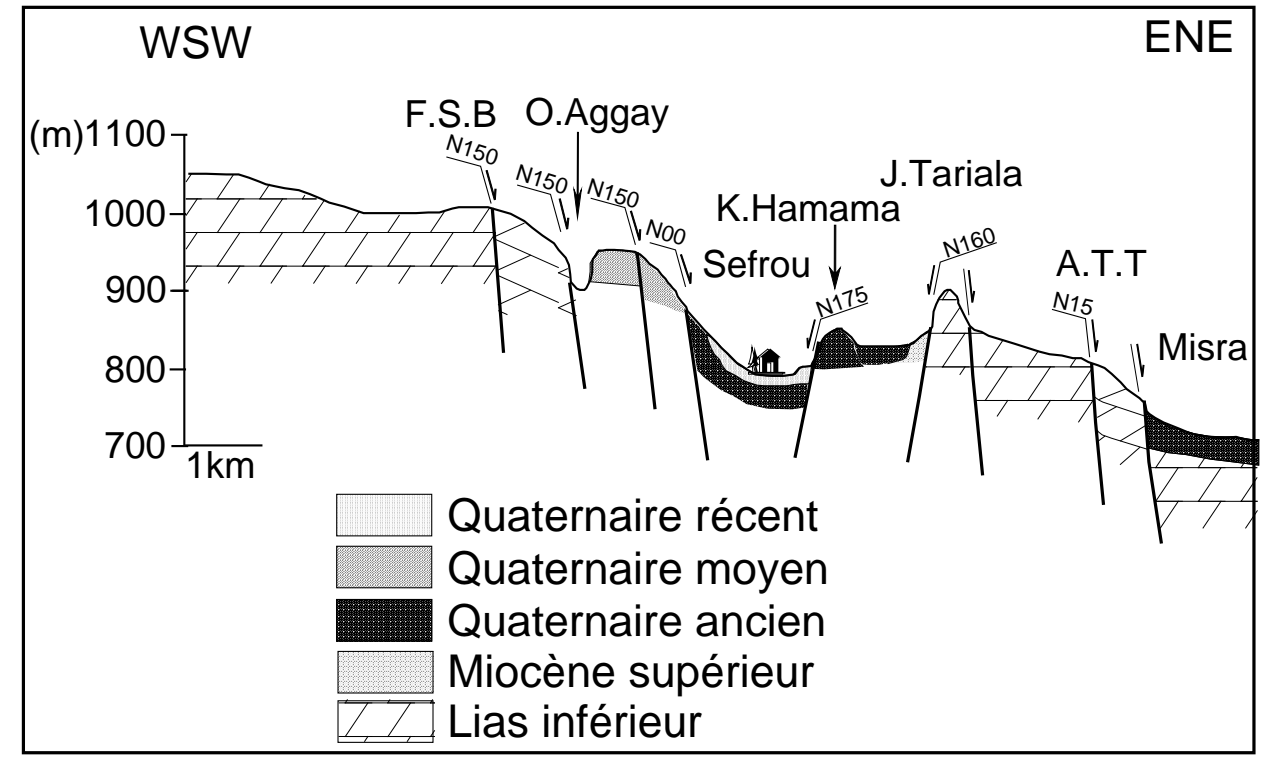

Figure 6 : Coupe structurale le long du bassin de Sefrou montrant le jeu normal des failles subméridiennes au Quaternaire (situation de la coupe : voir Fig. 2).

\section{Relation entre la fracturation et la répartition des sources d'eaux}

Généralement, les relations entre les différentes sources d'eaux, la nature des formations ainsi que la fracturation s'avèrent évidentes. En effet, la circulation des eaux en surface et en profondeur est tributaire des zones de faiblesses d'origine tectonique.

$\mathrm{Au}$ niveau du bassin de Sefrou, on constate que les principales sources sourdent soit à proximité des contacts anormaux (failles inverses, normales et/ou décrochantes), soit au niveau des nœuds tectoniques (point de rencontre de deux ou plusieurs fractures, failles ou accidents). C'est ainsi que notre étude menée dans ce sens, a permis de mettre en évidence la relation qui existe entre les différentes sources d'eaux et la fracturation.

Pour l'étude et la compréhension de cette relation, nous nous sommes basés sur quelques exemples qui représentent les principales sources du bassin de Sefrou. On distingue deux types de sources (El Fellah Idrissi et al., 2007) :

- source d'émergence : exemple la source Slama (Fig. 7),

- source de déversement : exemple la source Ghzale, Ghaba et Sidi Abdellah (Fig. 8, 9 et 10). 


\section{La source Slama}

La source Slama est située près de Bhalil, sur le flanc NW du bassin de Sefrou (Fig. 7). Elle contribue à l'alimentation en eau potable ainsi qu'à l'irrigation de quelques parcelles agricoles. Cette source prend naissance au pied de Jbel Mimet, dans la zone de passage vers la plaine de Saïs. Elle sourde au niveau du point d'intersection de deux failles, l'une subméridienne et l'autre orientée N70. L'émergence de la source Slama se fait dans les basaltes à inter - bancs carbonatés du Trias-Lias inférieur. Des paléo-éboulis de dolomies liasiques bordent la source des côtés est et ouest. Ces paléoéboulis d'âge Néogène à actuel (Charrière, 1989), sont le résultat des événements tectoniques qui ont affecté le flanc NW du bassin de Sefrou.

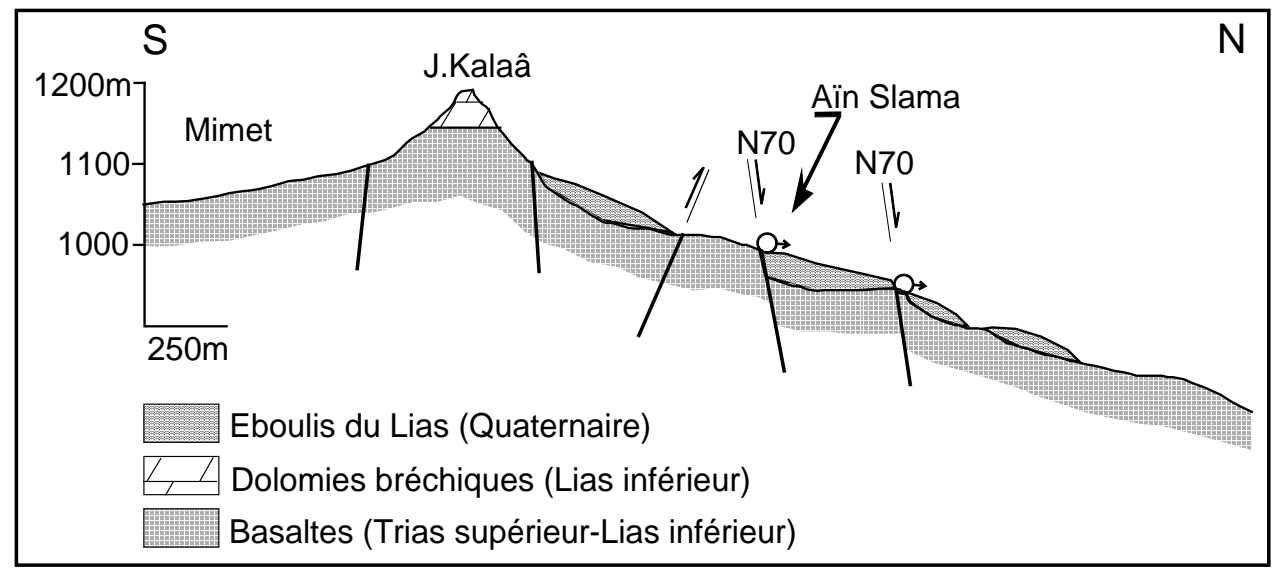

Figure 7 : Contexte géologique de la source Slama (situation de la coupe voir Fig. 2).

La source Slama est alimentée par la nappe triasico-liasique de Mimet. Cette nappe localisée dans la tranche superficielle des basaltes (partie altérée), donne naissance à de nombreuses sources au niveau de la zone de Mimet (sources Lemrouj, El Allig,...) et sur le flanc NW (sources Slama, Dik,...). L'émergence de ces sources est favorisée surtout par la diminution de la perméabilité au niveau de la partie altérée des basaltes doléritiques. Cette diminution se fait du haut en bas et sur une faible épaisseur (Dekayir et al., 2002).

À Mimet, les eaux souterraines de la nappe tiasico-liasique se partagent en deux parties : une partie s'écoule vers l'Est et le SE alimentant le bassin de Sefrou, et l'autre partie s'écoule vers le Nord et le NW, sur le flanc NW de ce bassin (Fig. 8). Ces écoulements souterrains suivent les pendages des couches et la direction des effondrements des blocs tectoniques. En effet, au Nord de Mimet la nappe coule suivant la direction de l'effondrement provoqué par le passage des failles normales N70 d'âge Pliocène moyensupérieur, ce qui favorise l'alimentation de la source Slama (cf. Fig. 7). 


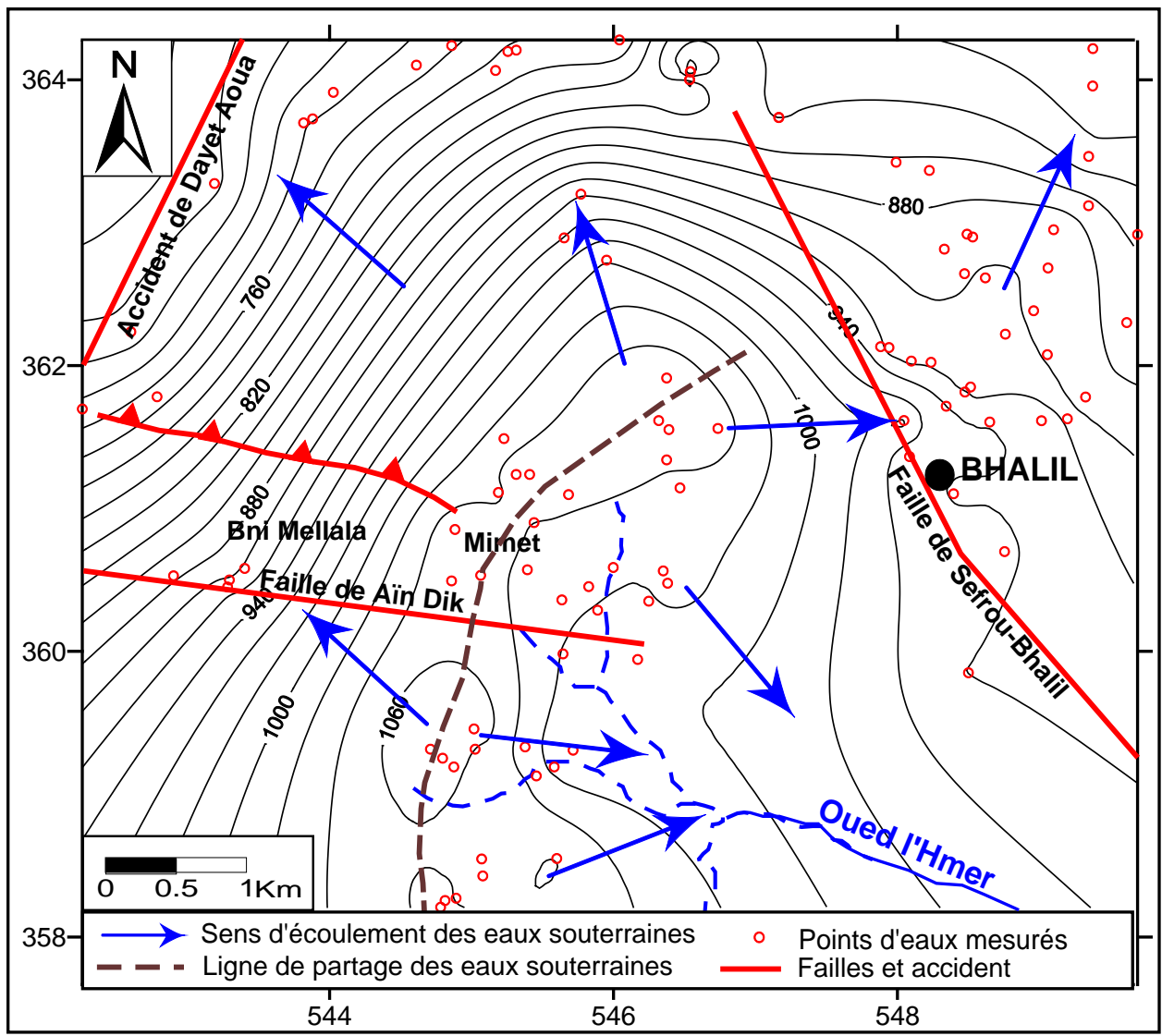

Figure 8 : Carte piézométrique de la nappe d'eau triasico-liasique de Mimet - Bhalil (Septembre2003).

\section{La source de Ghzal}

La source Ghzal est située à l'extrémité NW du plateau de Bhalil (cf. Fig. 2). Elle prend naissance au pied de la partie orientale de Jbel Ksiksou. Elle sourd au niveau de la zone de recoupement de l'une des failles N60 avec celle de Sefrou - Bhalil orientée N140. Il s’agit d'une source de déversement située à la base des dolomies liasiques au contacte des argiles rouges triasicoliasiques (Fig. 9). Les éboulis dolomitiques masquent la résurgence principale de la source qui apparaît à quelques mètres de celle-ci à la surface des basaltes triasico-liasique.

L’alimentation de la source est assurée par les eaux qui transitent du compartiment soulevé de Mimet vers le compartiment affaissé de Bhalil. En effet, les affleurements basaltiques de Mimet sont en relation hydraulique avec les dolomies de l'unité de Jbel Ksiksou. La nappe dans ces dolomies est alimentée par la pluviométrie et par abouchement avec les eaux des basaltes doléritiques. Cet abouchement est facilité par les fissures et les failles N60 affectant ces formations. 


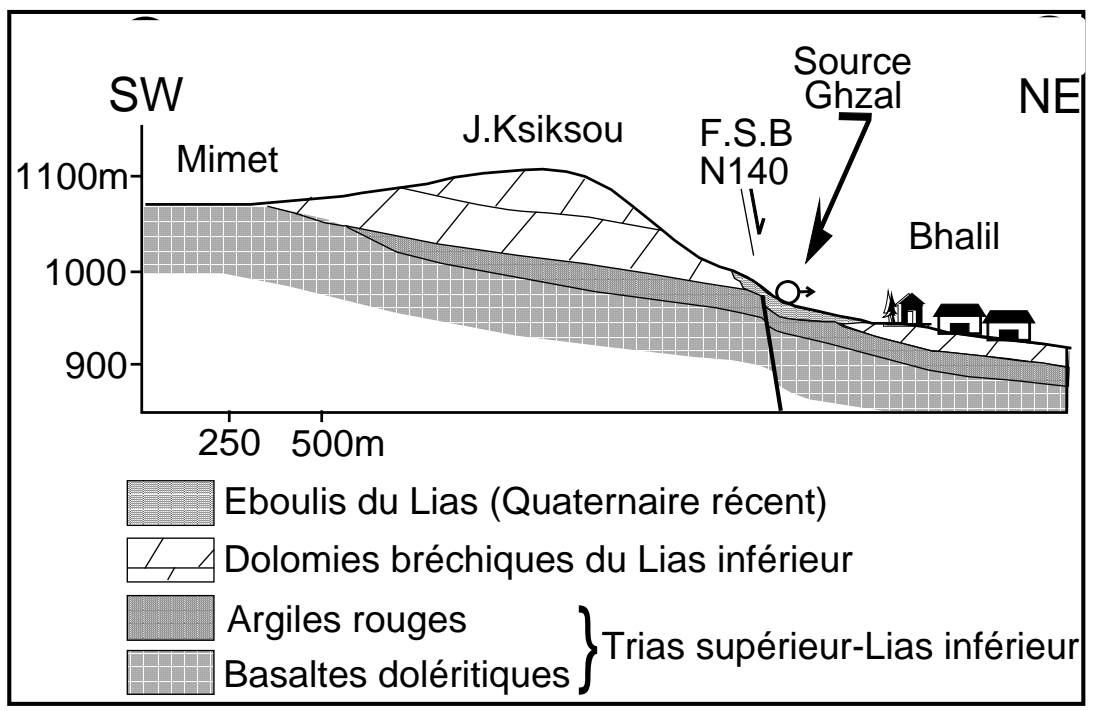

Figure 9 : Contexte géologique de la source Ghzal (situation de la coupe : voir Fig. 2).

\section{La source de Ghaba}

La source Ghaba est située au SE de Bhalil. Avec un très faible débit, les eaux de la source servent pour des utilisations domestiques. Elle prend naissance dans la zone de passage d'une faille normale N130 (cf. Fig. 2). La source Ghaba sourd à la base des poudingues de Skoura au contacte des argiles rouges triasico-infra liasiques (Fig. 10). Ces poudingues d'âge Pliocène inférieur-moyen (Charrière, 1990 ; Sabaoui, 1998 ; Hinaje, 2004), sont surmontés par des tufs et des conglomérats travertineux. L'ensemble de ces formations recouvrent en discordance angulaire mineure les dolomies liasiques et les argiles rouges triasico-infra liasiques qui se présentent au niveau de la source, en contacte directe avec cet ensemble. Un niveau pelliculaire argilo-marneux de la formation de "Harira " d'âge infraLiasique (Charrière, 1990) recouvre les argiles rouges.

L'alimentation de la source Ghaba est assurée par la nappe aquifère des dolomies liasiques. En amont de la source, les écoulements de la nappe s'effectuent de l'Ouest vers l'Est en direction du compartiment affaissé par la faille N130. Au cours de leur cheminement, les eaux de la nappe se déversent dans les basaltes et les dolomies du compartiment effondré. Ceci peut s'effectuer soit par abouchement directe avec l'aquifère, soit par l'intermédiaire des calcarénites messiniennes (cas de la source de Sidi Abdellah) et des poudingues de Skoura (cas de la source Ghaba). 


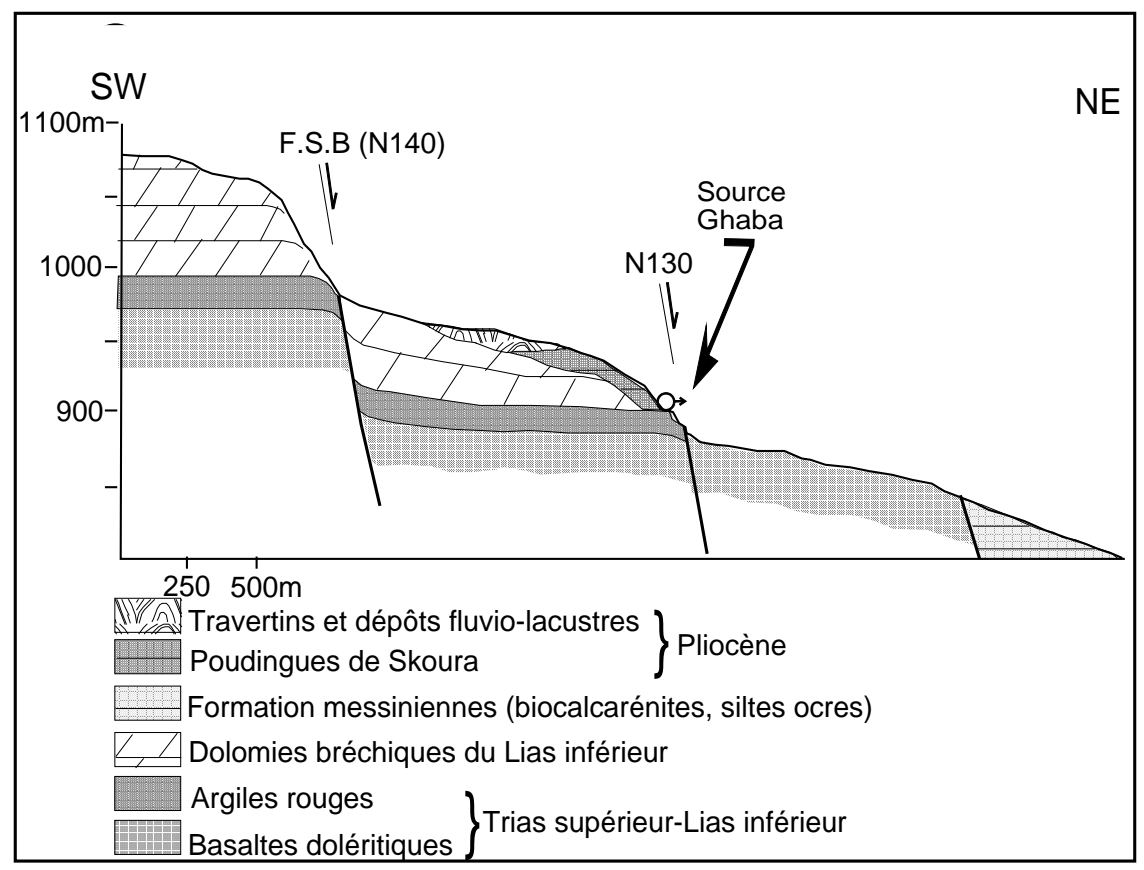

Figure 10 : Contexte géologique de la source Ghaba (situation de la coupe : voir Fig. 2).

La nature bréchique de l'aquifère liasique et l'absence d'un recouvrement imperméable ainsi que la position de la source Ghaba en aval de Bhalil traduisent l'importance de la vulnérabilité de cette source vis-à-vis de la pollution. Les causes de la pollution sont représentées surtout par l'existence des fosses septiques et du cimetière de Bhalil où pied duquel sourd la source Ghaba.

Les analyses effectuées sur les eaux de cette source (Tab.1) montrent une faible conductivité de celle-ci, ce qui reflète une faible minéralisation due à la faible concentration des eaux en éléments chimiques : chlorures, magnésium, bicarbonates, sodium, potassium, sulfates, et manganèse. L'élévation des teneurs en eaux du calcium et des nitrates est due à la contamination de la nappe par les eaux usées et à l'élevage.

Tableau 1 : Analyses physico-chimiques des eaux de la source Ghaba (Décembre 2004).

\begin{tabular}{|c|c|c|c|c|c|c|c|c|c|c|c|c|}
\hline $\begin{array}{c}\mathrm{T} \\
\left({ }^{\circ} \mathrm{C}\right)\end{array}$ & $\begin{array}{c}\text { Cond } \\
(\mu \mathrm{s} / \mathrm{cm})\end{array}$ & $\mathrm{pH}$ & $\begin{array}{c}\mathrm{Cl}^{-} \\
(\mathrm{mg} / \mathrm{l})\end{array}$ & $\begin{array}{c}\mathrm{C}^{2+} \\
(\mathrm{mg} / \mathrm{l})\end{array}$ & $\begin{array}{c}\mathrm{Mg}^{2+} \\
(\mathrm{mg} / \mathrm{l})\end{array}$ & $\begin{array}{c}\mathrm{HCO}^{3-} \\
(\mathrm{mg} / \mathrm{l})\end{array}$ & $\begin{array}{c}\mathrm{NO}_{3}{ }^{2-} \\
(\mathrm{mg} / \mathrm{l})\end{array}$ & $\begin{array}{c}\mathrm{SO}_{4}{ }^{2-} \\
(\mathrm{mg} / \mathrm{l})\end{array}$ & $\begin{array}{c}\mathrm{PT} \\
(\mathrm{mg} / \mathrm{l})\end{array}$ & $\begin{array}{c}\mathrm{Mn}^{2+} \\
(\mathrm{mg} / \mathrm{l})\end{array}$ & $\begin{array}{c}\mathrm{K}^{+} \\
(\mathrm{mg} / \mathrm{l})\end{array}$ & $\begin{array}{c}\mathrm{Na}^{+} \\
(\mathrm{mg} / \mathrm{l})\end{array}$ \\
\hline 16.4 & 851 & 7.56 & 63,9 & 122,4 & 9,12 & 240,34 & 131,08 & 14 & 0,416 & 0,48 & 2,35 & 22 \\
\hline
\end{tabular}




\section{La source de Sidi Abdellah}

La source Sidi Abdellah est située à environ 1,5km au NE de Bhalil (cf. Fig. 2). Elle est caractérisée par un très faible débit. Cette source sourd au niveau d'un nœud tectonique correspondant au recoupement de deux failles normales. Il s'agit de la faille normale N170 d'âge Quaternaire moyenrécent, qui recoupe une faille transversale N90 d'âge Pliocène moyensupérieur. La source prend naissance à la base des calcarénites tortonomessiniennes, qui recouvrent un substratum argileux triasico-liasique (Fig. 11). Ces calcarénites sont affectées par des fractures de même direction que la faille N90. Ces fractures contribuent donc au cheminement des eaux de la nappe liasique qui s'écoule, à travers les plans des failles subméridiennes, de l'Ouest vers l'Est perpendiculairement à celles-ci.

Au niveau de Bhalil la nappe circule dans les dolomies liasiques et dans les basaltes triasico-liasiques. Elle se dégorge au niveau des calcarénites où résurgent la source de Sidi Abdellah, qui correspond à une source de déversement. En effet, les calcarénites reposent en discordance angulaire mineure à la fois sur les argiles rouges et les dolomies (Fig.11). En aval de la source, dans les compartiments effondrés par les failles subméridiennes, la nappe continue à circuler au niveau des dolomies liasiques, de l'Ouest vers l'Est, et ensuite du Sud vers le Nord.

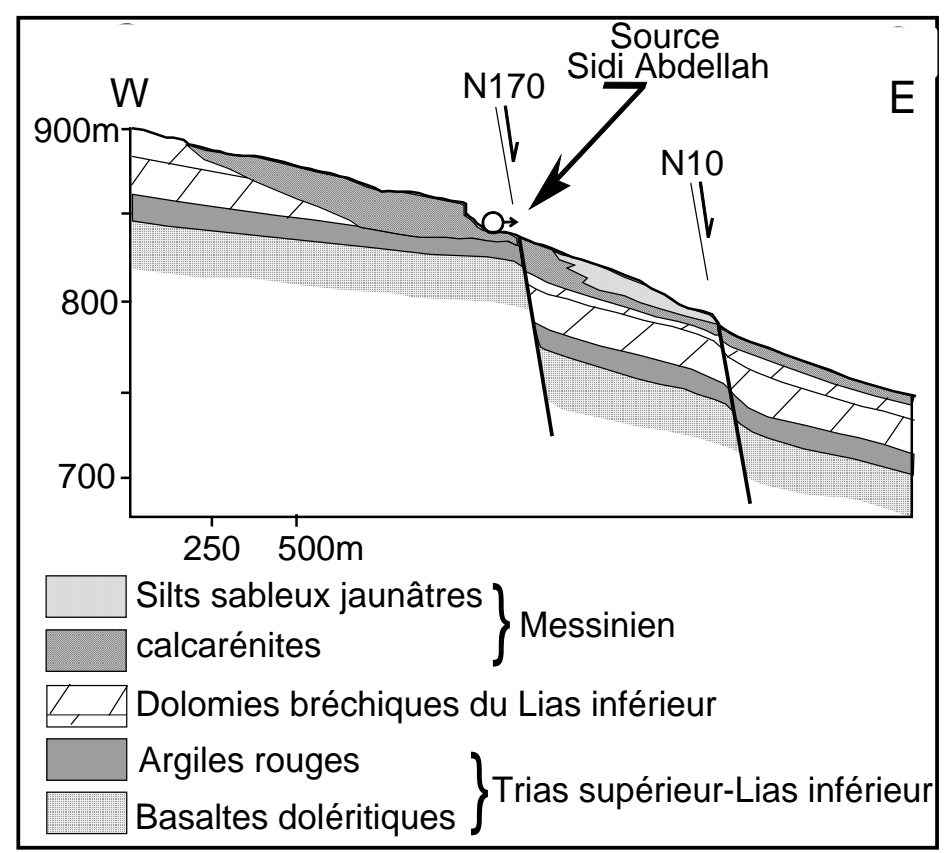

Fig. 11 : Contexte géologique de la source Sidi Abdellah (situation de la coupe : voir Fig.2). 
La source de Sidi Abdellah comme celle de Ghaba, est vulnérable à la pollution. Particulièrement par les eaux usées déversées directement dans les fosses septiques des villas de la zone de Msila qui fait partie de la ville de Bhalil.

\section{Conclusion}

La genèse du bassin hydrogéologique de Sefrou est liée à quatre principaux épisodes extensifs appartenant aux phases tectoniques d’âge Miocène, Pliocène et Quaternaire. Ces épisodes ont donné naissance à des failles orientées N130, N60 à N80, N30 et N170 responsables de la structure du bassin. Celles-ci jouent un rôle primordial dans l'organisation spatiale des écoulements d’eaux souterraines et superficielles. On constate que :

- le déplacement généralisé de la nappe du Sud vers le Nord dépend de la porosité des dolomies, de la fracturation et du compartimentage tectonique provoqué essentiellement par le jeu normal des failles N130 ;

- les failles normales subméridiennes constituent des axes et des conduits préférentiels pour le drainage des eaux souterraines vers le Nord ;

- les grabens constituent des couloirs permettant le rassemblement et le transfert d'importante quantité d'eau, tandis que les horsts et les plans de failles colmatés par les injections argileuses constituent des barrières naturelles provoquant le blocage et la réorientation de l'écoulement des eaux ;

- les principales sources d'eaux se répartissent à la faveur des zones de failles. Elles sont représentées par les sources d’émergence et les sources de déversement ;

\section{References :}

Aït Brahim, L. (1992). Tectonique cassante et état de contraintes récents du Maroc Nord, contribution à l'étude du risque sismo-tectonique au Maroc. Thèse d'Etat, Université Mohamed V - Rabat, 300 p.

Baudelot, S., Colin, J.P., \& Ouarhache, D. (1986). Le niveau sédimentaire associé aux basaltes triasiques sur la bordure septentrionale du causse d'El Hajeb (Maroc): données palynologiques et micropaléontologiques (Ostracode). Revue de Paléobiologie de Genève, vol. 5, n 2, p. 281-287.

Bentayeb, A., \& Leclerc, C. (1977). Ressources en eau du Maroc. Domaines atlasiques et sub-atlasique. Notes et Mémoires du Service Géologique ; Maroc, Tom 3, n²31. pp :66-89.

Biron, P. E., \& Courtinat, F. (1982). Contribution palynologique à la connaissance du Trias du Haut Atlas de Marrakech, Maroc, Geobios, 15, 1982, p. 231-235.

Bruel, T., Pierre Petit, J., Massonnat, G., Guerin, R., \& Nolf, J.L. (1999). Relation entre écoulement et fracture ouvertes dans un système aquifère 
compartimenté par des failles et mise en évidence d’une double porosité de fractures. Bulletin de la Société de Géologie. France, t.170, n³, 401-412.

Cacas, M.C., \& De Marsily, G. (1987). Modelling of flow through fractured rocks.- centre d’informatique géologique, Ecole des Mines de Paris, conférence (inédit).

Castany, G. (1982). Principes et méthodes de l’hydrogéologie. Bordas, Paris, 238p.

Charrière, A. (1990). Héritage hercynien et évolution géodynamique alpine d'une chaîne intracontinental : le Moyen Atlas au SE de Fès (Maroc), Thèse de Doctorat d'Etat, Toulouse, 589p.

Cousminer, H. L., \& Manspeizer, W. (1976). Triassic pollen date Moroccan High Atlas and the incipient rifting of Pangea as middle Carnien. Science, 191, pp. 943-945.

Dekayir, A., Danot, M., Allali, N. (2002). Apport des phyllosilicates dans la différenciation entre altération hypogène et altération supergène dans le basalte triasique du Moyen Atlas (Maroc), Comptes Rendu Géoscience, vol 334, pp. 877- 884.

El Fellah Idrissi, B., Hinaje, S., \& Lahrach, A. (2005). Influence de la tectonique cassante sur les caractéristiques hydrogéologiques des réservoirs du Causse de Sefrou (Moyen Atlas, Maroc). $18^{\text {ème }}$ Colloque International des Bassins Sédimentaires Marocains, Fès.

El Fellah Idrissi, B., Hinaje, S., \& Lahrach, A. (2007). Rôle hydrogéologique des fractures et leur influence sur la répartition des ressources en eau : cas des bassins de Sefrou et de l'Anoceur (Moyen Atlas, MAROC). $4^{\text {ème }}$ Journées Internationales des Géosciences de l’Environnement. Faculté des Sciences, Tétouan.

Hinaje, S. (2004). Tectonique cassante et paléochamps de contraintes dans le Moyen Atlas et le Haut Atlas central (Midelt - Errachidia) depuis le Trias jusqu’à l'actuel. Thèse Doctorat d'Etat, Université Mohamed V, Rabat. 363 p.

Hinaje, S., Aït Brahim, L., \& Gourari, L. (2002). Rôle de la tectonique cassante polyphasée (du Miocène supérieur à l'actuel) dans la genèse du tracé hydrographique de la vallée de l'Oued Aggay et l'effondrement du bassin de Sefrou (bordure NW du Moyen-Atlas, Maroc). Africa Geoscience Review, Vol. 9, N$^{\circ}$ 2, pp : 171-181.

Loiseau, P. (1987). Etude structurale et géostatistique des gneiss de la région du Cézallier (Massif central français). Modélisation tridimensionnelle de réseaux de fractures. Application à l'écoulement des fluides. Thèse de Doctorat de $3^{\text {ème }}$ cycle. Université d'Orléans.

Margat, J. (1952). Notes inédit sur l'hydrogéologie des Causses, C.E.H.

Pusch, R., \& Hokamark, H. (1991). Characterization of nearfield rok- Abasis for comparaison of repository cocepts.- SKB technical report, 92-06. 
Sabaoui, A. (1987). Structure et évolution alpine du Moyen-Atlas septentrional sur la transversale Tleta des Zerarda -Merhraoua (Sud Ouest de Taza, Maroc). Thèse de $3^{\text {ème }}$ cycle, Toulouse, $189 \mathrm{p}$.

Sabaoui, A. (1998). Rôles des inversions dans l'évolution méso-cénozoïque du Moyen-Atlas Septentrional (Maroc). L'exemple de la transversale El Menzel - Ribat Al Khayr - Bou Iblane. Thèse Doctorat d’Etat, Rabat, 410 p. Sabaoui, A., \& Hinaje, S. (2000). Le Néogène du Moyen Atlas : évolution tectono- sédimentaire, paléogéographie superposée et essai de corrélation avec le couloir sud-rifain. XI congress of regional committee on mediterranean neogene stratigraphy.

Taltasse, P. (1952). Notes inédit sur l'hydrogéologie des Causses. C.E.H. Services Géologique du Maroc, (1989). Carte géologique du Maroc "Sefrou". Notes et Mémoire, N 354. 